\title{
Simulation-Based Controller Design for an Active Auxiliary Bearing*
}

\author{
Lucas GINZINGER ${ }^{* *}$ and Heinz ULBRICH** \\ ** Institute of Applied Mechanics, Technische Universität München \\ 85748 Garching, Germany \\ E-mail: ginzinger@amm.mw.tum.de
}

\begin{abstract}
A framework for the development of a feedback controller for an active auxiliary bearing is presented. In a new approach, a dry bushing type auxiliary bearing is attached to the foundation via two unidirectional actuators. The control force is applied indirectly using the active auxiliary bearing only in case of rubbing. A simulation for the elastic rotor and the auxiliary bearing including the non-smooth nonlinear dynamics of the rubbing contact is used to develop the feedback controller. A robust two-phase control strategy has been developed which stabilizes the rotor system in case of rubbing and effectively avoids "backward whirling". Experimental studies have been carried out at a rotor test rig. The experiments show the outstanding success of the strategy. In case of rubbing, the contact forces are reduced up to $85 \%$.
\end{abstract}

Key words : Rotor Rubbing, Active Auxiliary Bearing, Feedback Control

\section{Introduction}

Rubbing phenomena in rotating machinery is a serious malfunction which can lead to the complete destruction of a system. In rotating machinery, increased efficiency is often achieved by tightening operation clearances. If the machine is not operating under normal conditions, the stationary and rotating elements are in danger of coming in contact. Conventional auxiliary bearings or touch down bearings are used to prevent direct contact between the rotor and the casing in case of a failure of magnetic bearings or when the rotor response is too large, but they do not stabilize the rotor. Various states of rotor rubbing, including the destructive backward whirling and very high impact forces are possible. In literature there are many publications about active vibration control of flexible rotors. Numerous works over the last 30 years deal with control forces acting directly on the rotor without contact using magnetic bearings, e.g. Refs. (7) and (10). The main disadvantage of this concept is the limited available space for the actuators. Therefore, concepts have been developed to actuate on the bearing housings and thereby indirectly on the rotor in opposition to magnet bearings, e.g. Refs. (3) and (6).

\section{Active Auxiliary Bearing}

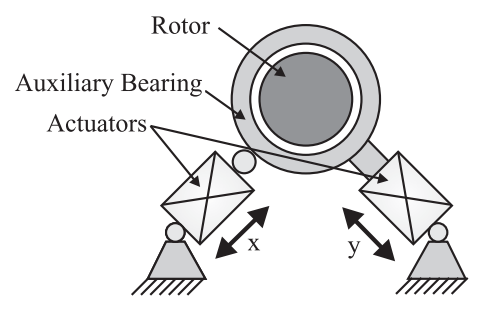

Fig. 1 Active auxiliary bearing

A simple rotor system is taken as an example to show the possibilities of an active auxiliary bearing to control the rubbing of a rotor. The control force is applied indirectly by a 
dry bushing type auxiliary bearing which is attached to the foundation via two unidirectional actuators as shown in Fig. 1. The actuators are mounted in a 90 degree alignment. For experimental verifications electromagnetic actuators have been used which have been developed at the Institute of Applied Mechanics, TU Munich. The main advantage of this type of electromagnetic actuator is a large stroke up to $1 \mathrm{~mm}$ at frequencies up to $250 \mathrm{~Hz}$. Forces up to $1000 \mathrm{~N}$ are available. Depending on the application of an active auxiliary bearing, the requirements for the actuators vary strongly. The selection of suitable actuators depends on various parameters, such as the size of the air gap in the auxiliary bearing and the rotational speed of the rotor system. The advantages of this control concept are the following: If the rotor system runs in the usual operation state, the active auxiliary bearing does not take effect, so the original design of the rotor system can be kept to largest extent unchanged. In case of a rotor to stator contact, the auxiliary bearing does not only limit a too large response amplitude of the rotor and prevents the rotor/blades and the casing/seals from direct contact, but also effectively reduces the rubbing severity and especially avoids the occurrence of destructive rubbing states such as backward whirling. The capability of existing auxiliary bearings, i.e. as safety bearings in active magnetic bearing systems or as run-through resonance support, can be well extended from this concept by introducing active control.

\section{Feedback Control}

- Center of the auxiliary bearing without control

+ Center of the auxiliary bearing (controlled)

........ Position of the auxiliary bearing without control Position of the auxiliary bearing (controlled)
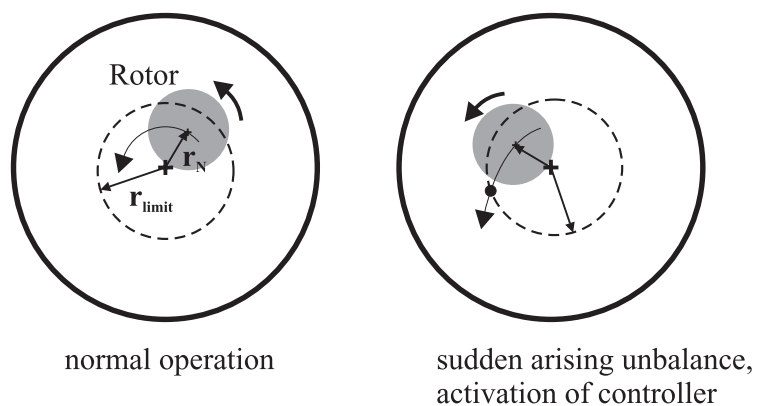

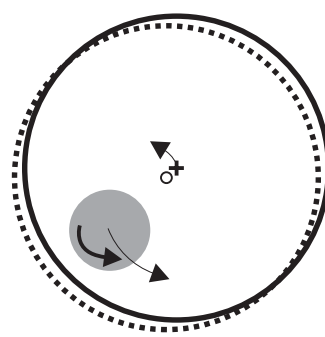

$1^{\text {st }}$ control phase, synchronisation

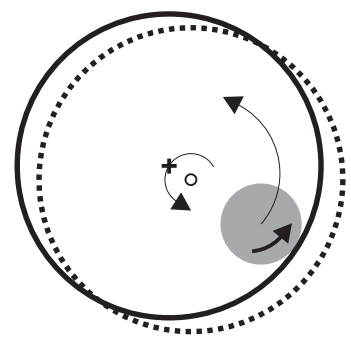

$2^{\text {nd }}$ control phase, permanent contact

Fig. 2 Principle of the control concept

A two-phase control strategy has been developed, which guarantees a smooth transition from free rotor motion to the state of full annular rub. To keep the principal purpose in mind, the control scheme also has to limit the rotor amplitude as a passive auxiliary bearing does. As input the controller only needs the position of the auxiliary bearing, the position of the rotor shaft inside the auxiliary bearing and the information of the shaft encoder. The operation phases of the control concept are shown in Fig. 2. During normal operation condition, which means that the rotor deflection $\mathbf{r}_{N}$ is smaller than a defined limit $\mathbf{r}_{\text {limit }}$, the controller is deac- 
tivated. When the rotor deflection becomes too large, e.g. due to a sudden arising unbalance, and $\mathbf{r}_{N}$ exceeds $\mathbf{r}_{\text {limit }}$, the controller is activated. In the first control phase, the movement of the auxiliary bearing is synchronized with the rotor orbit followed by a smooth transition to a contact. In the second phase, the feedback control assures a permanent contact in the rubbing state of "synchronous full annular rub" and low contact forces.

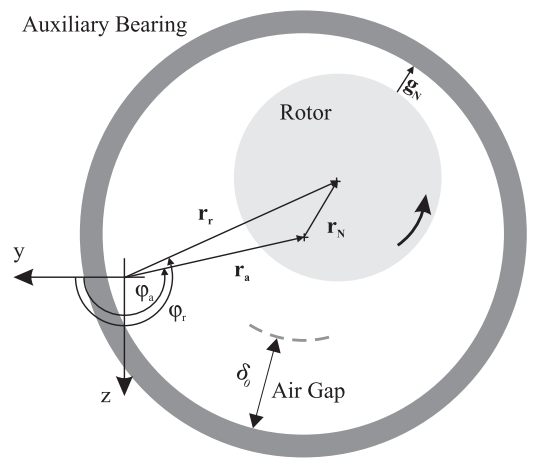

Fig. 3 Contact kinematics

In order to define the essential geometrical variables a cross section of the auxiliary bearing and the rotor is shown schematically in Fig. 3. The origin of the coordinate system coincides with the center of the undeformed rotor, $\mathbf{r}_{r}$ is the position vector to the center of the deformed rotor (in the cross section) and $\mathbf{r}_{a}$ to the center of the auxiliary bearing. The air gap in the auxiliary bearing is $\delta_{0}$ and $\mathbf{r}_{N}$ represents the vector from the center of the auxiliary bearing to the center of the rotor. Additionally the polar angles $\varphi_{a}$ and $\varphi_{r}$ of the vectors $\mathbf{r}_{a}$ and $\mathbf{r}_{r}$ are introduced.

The overall control target can be specified as

$$
\mathbf{q}_{a d}=\underset{(\ddot{\mathbf{q}}, \mathbf{q}, \mathbf{q})}{\arg \min }\left\{\begin{array}{l}
g_{N} \\
\dot{g}_{N}
\end{array},\right.
$$

with $\arg$ min $=$ values of $\ddot{\mathbf{q}}, \dot{\mathbf{q}}, \mathbf{q}$ that minimizes $g_{N}$ and $\dot{g}_{N}$. The vector $\mathbf{q}$ are the generalized coordinates of the rotor and the auxiliary bearing, $g_{N}$ is the distance between the contact points and $\mathbf{q}_{a d}$ the target trajectory for the auxiliary bearing. Equation (1) includes that the relative distance becomes zero to get a permanent contact and the relative velocity in normal direction should be as small as possible to get a smooth transition from free rotor motion to the state of full annular rub. The relative velocity of the contact point in tangential direction $\dot{g}_{T}$ will not be taken in consideration in Eq. (1) because a non-sliding contact would cause a backward whirl, which is not wanted.

In both phases of the control scheme, the desired position of the auxiliary bearing is chosen in a way that the movement of the auxiliary bearing is synchronized with the orbit of the rotor and the contact point coincides with the point of the surface of the rotor which is farthest from the origin of coordinate system, Fig. 3. This means that

$$
\varphi_{\text {a desired }}=\varphi_{r}
$$

In case of contact this results in a movement pattern of "synchronous full annular rub". The destructive "backward whirl" is avoided.

Furthermore the desired polar radius $\mid \mathbf{r}_{N}$ desired $\mid$ is needed to determine the desired position of the auxiliary bearing according the equation

$$
\mathbf{r}_{a \text { desired }}=\left(\begin{array}{c}
\varphi_{a} \\
\left|\mathbf{r}_{a}\right|
\end{array}\right)=\left(\begin{array}{c}
\varphi_{r} \\
\left|\mathbf{r}_{r}\right|-\left|\mathbf{r}_{N \text { desired }}\right|
\end{array}\right) .
$$

So the $2 \mathrm{D}$ problem to control the orbit has turned into a $1 \mathrm{D}$ control problem of $\mid \mathbf{r}_{N}$ desired $\mid$. In the first control phase (free rotor motion), which means $\left|\mathbf{r}_{N}\right| \leq \delta_{0}$, the target position of the 
auxiliary bearing follows

$$
\begin{aligned}
& \left|\mathbf{r}_{N \text { desired }}\right|=\left|\mathbf{r}_{N}\right|-\int\left(\left|\dot{\mathbf{r}}_{N}\right|-\mathrm{v}_{\text {pmax }}\right) d t= \\
& =\left|\mathbf{r}_{N}\right|-\int\left(\left|\dot{\mathbf{r}}_{N}\right|-A e^{\alpha\left|\mathbf{r}_{r}\right|}\right) d t,
\end{aligned}
$$

where $\mathrm{v}_{\text {pmax }}$ is the maximum relative velocity of the contact points. The constant factors $A$ and $\alpha$ are chosen in such a way that first impact is kept small but also that the amplitude of the rotor does not exceed $\delta_{0}$, to meet the principal purpose of an auxiliary bearing.

In case of contact (second control phase), which means $\left|\mathbf{r}_{N}\right|=\delta_{0}$, the desired distance $\left|\mathbf{r}_{N \text { desired }}\right|$ follows:

$$
\left|\mathbf{r}_{N \text { desired }}\right|=\delta_{0}+\frac{1}{K_{P}} f_{\text {perm }},
$$

with $f_{\text {perm }}$ the desired contact force during the permanent contact and $\frac{1}{K_{P}}$ a conversion coefficient. Note that it is necessary to choose a desired contact force, which is large enough to ensure a permanent contact despite of elements of uncertainty of the measurement and control system.

A feedback linearization is used to control the electromagnetic actuators, see Refs. (4) and (8), which is based on a mechanical model of the active auxiliary bearing including the nonlinear dynamics of actuators and the joint friction. There are three main effects of the proposed control concept which improve the dynamics of a rubbing rotor system:

- The avoidance of multiple impacts reduces the whole load of the system significantly.

- The control concept introduces additional external damping into the rotor system because the controlled motion of the auxiliary bearing assures that the contact force in normal direction points towards the center of the undeformed rotor shaft, see Eq. (3). Additionally the friction force in tangential direction points in opposition to the rotational direction.

- As a consequence of the permanent contact between the rotor and the auxiliary bearing there is a change in the stiffness of the whole system. So it is possible to shift the resonant frequencies of a rubbing rotor running at a critical speed.

\section{Simulation Environment}

For the analysis of the dynamics of a controlled rubbing rotor system and the optimization of the feedback control system the simulation environment MBSim (http://mbsim.berlios.de), which was developed at the Institute of Applied Mechanics, is used. MBSim is based on a framework for the efficient simulation of multi-body-systems with unilateral contacts and elastic elements. The framework comprises the description of the system dynamics as well as numerical methods as provided in Refs. (2) and (11). A brief overview will be given in the following. For a more comprehensive introduction to the formulation and numerics of non-smooth dynamics see Refs. (1) and (9).

The non-smooth dynamics of the system is described in terms of a measure differential equation. The dynamics of a bi- and unilateral constrained system can be expressed by

$$
\mathbf{M} d \mathbf{u}=\mathbf{h} d t+\mathbf{W} d \mathbf{\Lambda} .
$$

The matrix $\mathbf{M}=\mathbf{M}(\mathbf{q})$ denotes the symmetric, positive definite mass matrix and depends on the $f$-dimensional vector of generalized coordinates $\mathbf{q} \in \mathbb{R}^{f}$. The vector $\mathbf{u}=\dot{\mathbf{q}}$ denotes the velocity vector. The acceleration measure

$$
d \mathbf{u}=\dot{\mathbf{u}} d t+\left(\mathbf{u}^{+}-\mathbf{u}^{-}\right) d \eta
$$

is the sum of the continuous part $\dot{\mathbf{u}} d t$ and the discrete parts $\left(\mathbf{u}^{+}-\mathbf{u}^{-}\right) d \eta$. The second term is the difference of the left and the right limit of the velocities weighted by the sum of the DIRAC 
delta functions $d \delta_{i}$ at the discontinuities $t_{i}$ :

$$
\begin{aligned}
& d \eta=\sum_{i} d \delta_{i}, \\
& d \delta_{i}=d \delta\left(t-t_{i}\right)=\left\{\begin{array}{c}
\infty \text { if } t=t_{i} \\
0 \text { if } t \neq t_{i}
\end{array},\right.
\end{aligned}
$$

On the right hand side of Eq. (7) the vector $\mathbf{h}=\mathbf{h}(\mathbf{u}, \mathbf{q}, t)$ contains all smooth external, internal and gyroscopic forces. The reaction measure in the contacts $\mathbf{W} d \mathbf{\Lambda}$ is decomposed by the generalized force directions $\mathbf{W}=\mathbf{W}(\mathbf{q})$ and the magnitudes $d \boldsymbol{\Lambda}$. In analogy to the acceleration measure, the reaction measure $d \boldsymbol{\Lambda}$ contains forces $\lambda$ due to persisting contacts as well as impulses $\boldsymbol{\Lambda}$ due to collisions of bodies at the impact times $t_{i}$ :

$$
d \mathbf{\Lambda}=\lambda d t+\Lambda d \eta
$$

Integrating Eq. (7) under consideration of the DIRAC delta (9) yields the classical equations of motion for a constrained system and the impact equations.

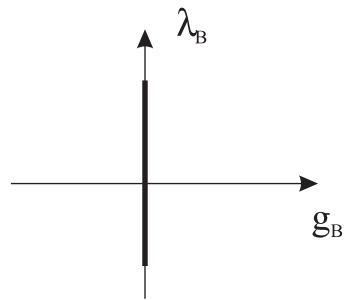

(a) Bilateral constraint

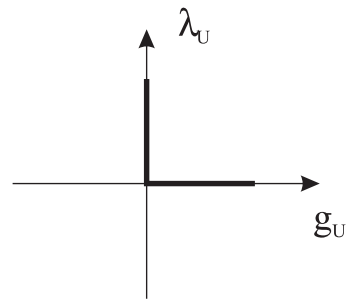

(b) Unilateral constraint

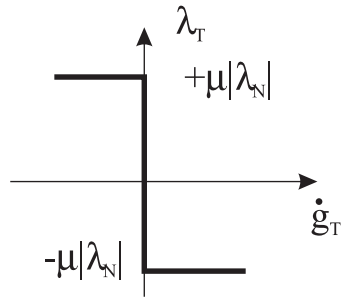

(c) COULOMB friction

Fig. 4 Force laws for bi- and unilateral contacts and friction

The computation of the accelerations $\dot{\mathbf{u}}$ as well as the post-impact velocities $\mathbf{u}_{i}^{+}$in Eq. (7) requires the knowledge of the unknown contact reactions $\lambda$ and $\boldsymbol{\Lambda}_{i}$, respectively. Thus, additional contact laws must be constituted. Contacts between bodies in the system are modeled as discrete point contacts whereby the contact zone is assumed to be totally rigid. Deformations of elastic components are only regarded in form of the overall discretization, no local deformation e.g. of a beam cross-section is modeled. Consequently, a contact corresponds to a constraint. In this context two different types of contacts are considered, for which different contact laws hold: persisting contacts which are always closed and contact that may be open or closed.

In the following, the force laws of the two contact types are explained for smooth and nonsmooth motion. For this, the contact reactions

$$
\begin{aligned}
& \mathbf{W} d \mathbf{\Lambda}=\left(\begin{array}{ll}
\mathbf{W}_{N} & \mathbf{W}_{T}
\end{array}\right)\left(\begin{array}{c}
d \boldsymbol{\Lambda}_{N} \\
d \boldsymbol{\Lambda}_{T}
\end{array}\right)= \\
& =\left(\begin{array}{lll}
\mathbf{W}_{B} & \mathbf{W}_{U} & \mathbf{W}_{T}
\end{array}\right)\left(\begin{array}{c}
d \boldsymbol{\Lambda}_{B} \\
d \boldsymbol{\Lambda}_{U} \\
d \boldsymbol{\Lambda}_{T}
\end{array}\right)
\end{aligned}
$$

are decomposed into components normal (index $\mathrm{N}$ ) - split up in bilateral (B) and unilateral (U) - and tangential (T) to the contact plane.

\section{Dynamics between Impacts}

First of all, only smooth motion is considered, i.e. no impacts occur. Then a bilateral contact implies a bilateral constraint of the form

$$
g_{B}=0, \lambda_{B} \in \mathbb{R},
$$


where $g_{B}$ denotes the normal distance of the interacting bodies in the contact point. The second type of contact also allows for detachment. The associated unilateral constraint is given by the SigNORINI-FICHERA-condition

$$
g_{U} \geq 0, \lambda_{U} \geq 0, \quad g_{U} \lambda_{U}=0
$$

The respective force laws are shown in Figs. 4 (a) and 4 (b).

For both bi- and unilateral constraints dry friction is considered. In order to establish Coulomb's law, the force of a single contact is decomposed in a component $\lambda_{N} \in\left\{\lambda_{B}, \lambda_{U}\right\}$ normal to the contact plane and - in case of three dimensional dynamics - tangential components $\lambda_{T}$ in friction direction. Using the relative tangential velocity $\dot{g}_{T}$, Coulomb's friction law is given by

$$
\begin{aligned}
& \dot{g}_{T}=0 \Rightarrow\left|\lambda_{T}\right| \leq \mu_{0}\left|\lambda_{N}\right| \\
& \dot{g}_{T} \neq 0 \Rightarrow \lambda_{T}=-\frac{\dot{g}_{T}}{\left|\dot{g}_{T}\right|} \mu\left|\lambda_{N}\right| .
\end{aligned}
$$

For the planar case, the force law of a tangential frictional contact is plotted in Fig. 4 (c).

\section{Impact dynamics}

In contrast to persisting and detaching contacts, a closing contact implies a discontinuity in the relative and therewith possibly all generalized velocities. Therefore impacts must be treated separately. The effect of an impact of a specific contact may concern all other constraints, the bilateral as well as the unilateral ones.

The impact law for a bilateral contact is given by

$$
\dot{g}_{B}^{+}=0, \Lambda_{B} \in \mathbb{R}
$$

and ensures that relation (14) is not violated after collisions. Given on impulsive level, NewTON's impact law in the formulation of MOREAU

$$
\dot{g}_{U}^{+} \geq 0, \Lambda_{U} \geq 0, \dot{g}_{U}^{+} \Lambda_{U}=0
$$

and Coulomb's friction law with the normal reaction $\Lambda_{N} \in\left\{\Lambda_{B}, \Lambda_{U}\right\}$

$$
\begin{aligned}
& \dot{g}_{T}^{+}=0 \Rightarrow\left|\Lambda_{T}\right| \leq \mu\left|\Lambda_{N}\right| \\
& \dot{g}_{T}^{+} \neq 0 \Rightarrow \Lambda_{T}=-\frac{\dot{g}_{T}^{+}}{\left|\dot{g}_{T}^{+}\right|} \mu\left|\Lambda_{N}\right|
\end{aligned}
$$

holds for active contacts with $g_{N}=0$ only.

\section{Elastic components}

The rotor shaft is modeled by a spatial bending-torsional beam using a polynomial finiteelement formulation for slender structures. Based on the EulER-BERnoulli theory with superposed torsion and small deflections, all deformations are described in rotating reference systems individually attached to each node. The formulation allows for arbitrary dynamic contact situations as introduced in the previous section, especially non-smooth dynamics including unilateral contacts and dry friction. For the present case, rigid disks are bilaterally bound to the shaft; the two unilateral contacts to the top and bottom circles of auxiliary bearing are modeled rigid including Coulomb friction.

\section{Numerical Framework}

The set of constrained measure differential equations is discretized and integrated by a half-explicit time-stepping method. The underline discretization is built with consideration of non-smoothness characterized by jumps in the system velocities. The evaluation of the force-laws is done by a fixed-point iteration scheme. A detailed description of the numerical framework can be found in Ref. (2). A comprehensive review on time integration of nonsmooth systems is provided in Ref. (9). 


\section{Cosimulation with Simulink}

A co-simulation between MBSim and MatLab/Simulink is used to integrate the feedback controller. MATLAB provides an Application Program Interface (API) called "MATLAB engine" which allows to call MatLaB from $\mathrm{C}$ as a computation engine. MatLaB engine programs communicates with a separate MATLAB process via pipes (in Unix) and through ActiveX on Microsoft Windows. There is a library of functions provided that allows you to start and end a process, send data to and from MatLAB, and send commands to be processed. Figure 5

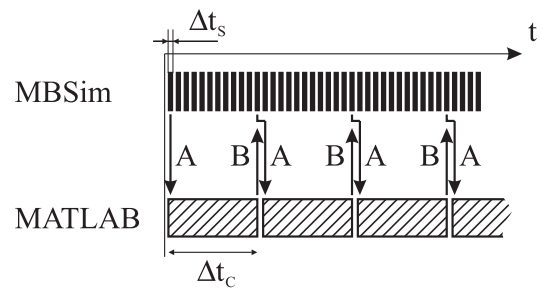

Fig. 5 Cosimulation

shows the synchronization of the cosimulation. The feedback controller is calculated in MATLAB/Simulink with a fixed time step size of $\Delta t_{C}$ using an Euler discretization as it is used at the experimental test rig. The simulation is calculated with the much lower fixed time step size $\Delta t_{S}$ of the time stepping integrator. The synchronization starts with "A". The positions and velocities, which are used by the feedback controller, are transferred to MATLAB and one calculation step of the controller is started in MAтLAB. In the meanwhile the multibody simulation MBSim calculates until the end of the controller time step $\Delta t_{C}$ is reached. With the synchronization "B" the calculated control force for the actuators is transferred to MBSim and the actuator force in the multibody simulation is updated.

The advantages of the co-simulation are the following. Since the dSpace real-time hardware uses Simulink for the code generation of the controller, a single Simulink model of the controller can be used for both - simulation and experiment. So a very rapid controller development and optimization can be achieved. There is also no error source caused by the modeling of the test rig controller in another simulation software. On the other hand, the cosimulation takes advantage of recent multi-core-processors, because between the synchronization steps MBSim and MATLAB are two independent processes.

\section{Modeling}

The simulation model of the rotor system represents the test rig and comprises the flexible rotor, a unilateral contact with friction between the rotor and the auxiliary bearing and a cosimulation between MBSim and MATLAB/SIMULINK to integrate the feedback controller. There are three rigid discs - the big disc, one small disc which is running inside the auxiliary bearing and a disc which is running inside the magnetic bearing. The discs are rigidly coupled on the elastic rotor. The rotor is attached to the environment via two spring-damper elements, which represents the ball bearings and bearing housings. The modeling of the auxiliary bearing includes the actuators, the three joints and the joint friction. The contact between the rotor and the auxiliary bearing is modeled as a rigid contact as described above. The overall model is a full 3D model of the presented rotor system.

\section{Controller Design}

The optimal parameters for the feedback controller for the test rig have been determined using the simulation. After this, experiments with these parameters have been carried out and the results have been used to verify the simulation. Using the verified simulation the parameters have been optimized again. A comparision of simulation results and experiments can be found in Ref. (5). 


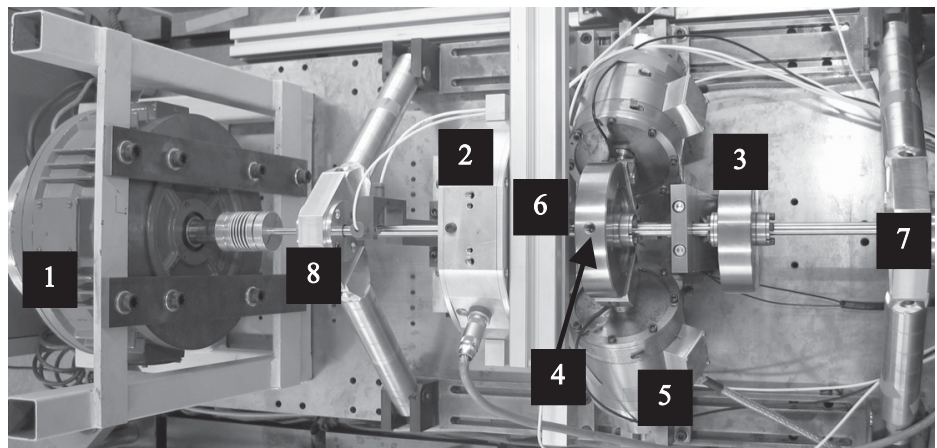

Fig. 6 Test rig (1: drive system, 2: magnetic bearing, 3: rotor disk, 4: auxiliary bearing, 5: electromagnetic actuators, 6: displacement sensors, 7: accelerometer, ball bearing, 8 : ball bearing)

\section{Test Rig}

The test rig can be assembled in various modifications. The assembly with a very flexible rotor shaft (diameter 12mm) is shown in Fig. 6 . The air gap between the rotor and the auxiliary bearing is $0.3 \mathrm{~mm}$. A magnetic bearing is used to create realistic excitations to the rotor such as a sudden arising unbalance, so that the rotor comes into contact with the auxiliary bearing. A direct current disc-servomotor allows a rotational speed up to $3500 \mathrm{rpm}$. Several sensors are used to gather information. There are two eddy current displacement sensors to measure the position of the rotor besides the auxiliary bearing. The same sensors are installed inside the actuators. Load washers in each actuator are measuring the actuator forces, from which the contact forces are determined indirectly. With the help of accelerometers the load of the bearings are recorded. The measured forces and accelerations are only used to show the performance of the control system.

\section{Experiments}

For the experiments a dSpace real-time hardware board ("DS1103") is used for the feedback controller, which main advantages are the very high flexibility and the processing power. For the analysis of the controller, the contact forces between rotor and the auxiliary bearing during rubbing are highly important. It is hardly possible to measure these forces directly without interfering with the whole system. Therefore they will be determined indirectly via measurements of the forces of the actuators and using the mathematical model of the system. Experimental results of rotor rubbing at the test rig with and without controlled auxiliary bearing are presented. For the case "without control" the auxiliary bearing was fixed mechanically

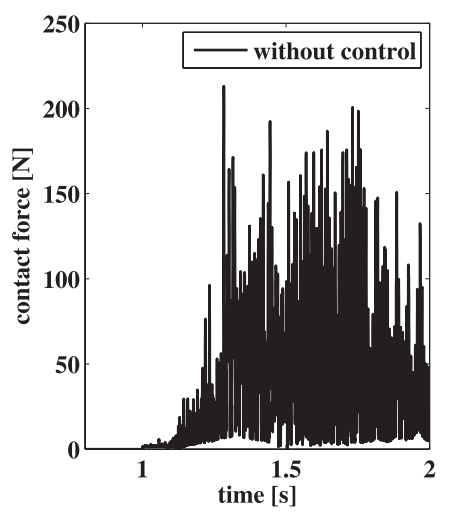

Fig. 7 Experimental results 1 - contact forces: the controller is DEACTIVATED

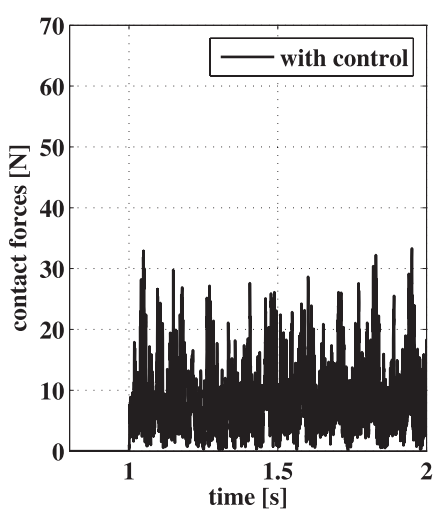

Fig. 8 Experimental results 2 - contact forces: the controller is ACTIVATED 


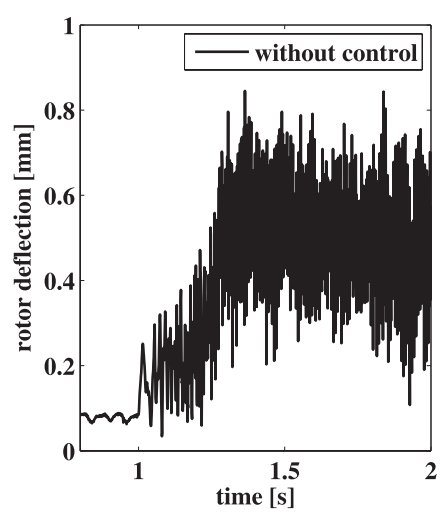

Fig. 9 Experimental results 1 - rotor deflection: the controller is DEACTIVATED

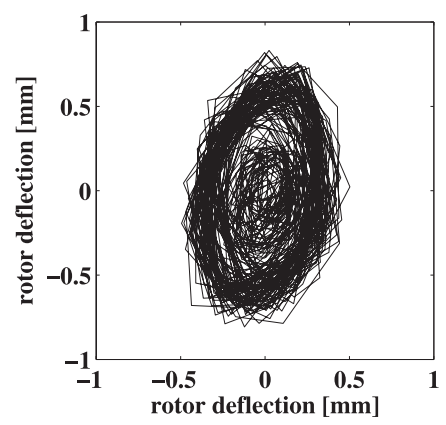

Fig. 11 Experimental results 1 - orbit: the controller is DEACTIVATED

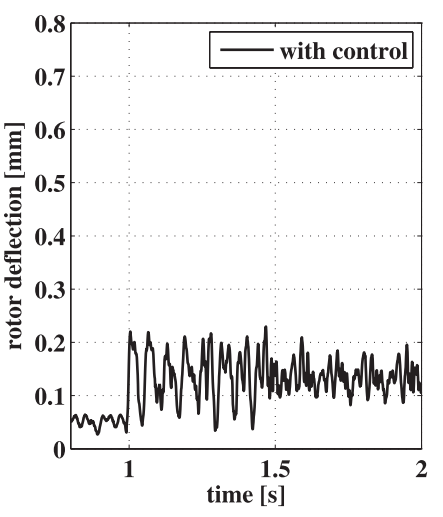

Fig. 10 Experimental results 2 - rotor deflection: the controller is ACTIVATED

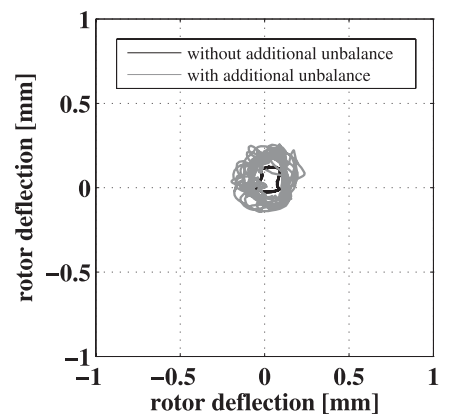

Fig. 12 Experimental results 2 - orbit: the controller is ACTIVATED

concentrically with the undeformed rotor and was working as a conventional passive auxiliary bearing.

The first results show an experiment without using the control system. The rotor system is running at a constant speed of $500 \mathrm{rpm}$ without additional unbalance. At a time of $t=1.0 \mathrm{~s}$ the magnetic bearing applies a sudden arising unbalance. Figure 7 shows the contact forces versus the time, Fig. 9 the rotor deflection versus the time and Fig. 11 the rotor orbit. Without the additional unbalance there is no contact between rotor and the auxiliary bearing. The rotor deflection is below $0.1 \mathrm{~mm}$. After the arising of the unbalance at $t=1.0 \mathrm{~s}$ the rotor comes into contact with the auxiliary bearing. After a time of $0.15 \mathrm{~s}$ the rubbing turns into the destructive "backward whirling" with very high contact forces and a large deflection.

The same experiment ( $500 \mathrm{rpm}$, additional unbalance after $t=1.0 \mathrm{~s}$ ) has been repeated with an activated control system. Figures 8, 10 and 12 show the measurements with the active auxiliary bearing. The automatic activation system tracks the position of the rotor and activates the control system after the arising of the unbalance at a time of $t=1.0 \mathrm{~s}$ just before the occurrence of the first contact. The control system is able to assure a permanent contact in the desired state of "synchronous full annular rub" with very low contact forces just after the arising of the unbalance. A state of "backward whirling" can be avoided. For the presented experimental results, the contact forces are reduced by $85 \%$. Please note the different scale of Fig. 8 compared to Fig. 7. At the same time the rotor deflection is reduced by $75 \%$. Figure 12 shows the rotor orbit during the experiment. The orbit before $t=1.0 \mathrm{~s}$ (without additional unbalance) is plottet black and the orbit during the controlled state of rubbing is plottet black.

It should be denoted, that there is no lubrication inside the auxiliary bearing in both experi- 
ments. This results in a very high friction, which is the "worst case" for the control system. In case of a lower friction coefficient the requirements to the control system are much lower and the rotor orbit during rubbing is smoother than the presented one.

\section{Conclusions}

A framework for the development of a feedback controller for an active auxiliary bearing is presented. Using the simulation of the elastic rotor and the auxiliary bearing a robust twophase control strategy has been developed. The controller assures a smooth transition from free rotor motion to the state of full annular rub with very low contact forces. Experimental studies have been carried out at a rotor test rig. A comparison of experiments with and without actively controlled auxiliary bearing shows the success of the control system. In case of rubbing, the contact forces are reduced up to $85 \%$ using the control system. At the same time the rotor deflection is decreased, too.

There are several possibilities of supporting a rotor system by using an active auxiliary bearing. In case of a suddenly arising unbalance, the controller is activated automatically if the rotor response is too large. The active control stabilizes the rotor and keeps the load of the whole system low, so that a safe coast-down is possible. On the other hand, it is also possible to use the control system for a safe run-through of a resonance frequency. Future research work will focus on the investigation of the relationship between the parameters of the rotor system and the requirements for the actuators to control the rubbing in an auxiliary bearing. Furthermore concepts for several possible applications will be developed.

\section{References}

( 1 ) B. Brogliato, A. A. Ten Dam, L. Paoli, F. Got, and M. Abadie. Numerical simulation of finite dimensional multibody nonsmooth mechanical systems. In ASME Applied Mechanics Reviews, 2002.

( 2 ) M. Förg, R. Zander, and H. Ulbrich. A framework for the efficient simulation of spatial contact problems. In Proceedings of the ECCOMAS Conference on Multi-Body Systems, Milano, Italy, 2007.

( 3 ) S. Fuerst and H. Ulbrich. An active support system for rotors with oil-film bearings. In 4th Int. Conf. on Vibrations in Rotating Machinery of the Institution of Mechanical Engineers, pages 61-68, Edinburgh, Scotland, 1987.

( 4 ) L. Ginzinger and H. Ulbrich. Feedback linearization rub control using an active auxiliary bearing. In 12th IFToMM World Congress, Besancon, France, 2007.

( 5 ) L. Ginzinger, B. Heckmann and H. Ulbrich. Simulation Environment for Rotor Systems with Unilateral Contacts and Active Elements In Proceedings of the XIII International Symposium on Dynamic Problems of Mechanics (DINAME 2009), Angra dos Reis, RJ , Brazil, March 2nd - March 6th, 2009.

( 6 ) J.W. Moore, D.W. Lewis, and J. Heinzmann. Feasibility of active instability problems in high-performance turbomachinery. In Proceedings of Workshop, Texas A $\mathcal{F} M$ University, College Station, Texas,USA, 1980.

( 7 ) G. Schweitzer. Stabilization of self-exited rotor vibration by an active damper. In $D y$ namics of Rotors - IUTAM Symposium, Lyngby,Denmark, 1974.

( 8 ) Jean-Jacques E. Slotine and Weiping Li. Applied Nonlinear Control. Prentice Hall, 1991.

( 9 ) Ch. Studer. Augmented time-stepping integration of non-smooth dynamical systems. In ETH E-Collection, 2008.

(10) H. Ulbrich, G. Schweitzer, and E. Bauser. A rotor supported without contact - theory and application. In Proceedings of the Fifth World Congress on Theory of Machines and Mechanisms, 1979.

(11) R. Zander, Th. Schindler, M. Friedrich, R. Huber, M. Förg, and H. Ulbrich. Non-smooth dynamics in academia and industry: recent work at tu münchen. In Acta Mechanica, 2008 . 